\title{
BCE: Berkeley's Common Scientific Compute Environment for Research and Education
}

\author{
Dav Clark ${ }^{\ddagger *}$, Aaron Culich ${ }^{\ddagger}$, Brian Hamlin ${ }^{\S}$, Ryan Lovett ${ }^{\ddagger}$ \\ http://www. youtube.com/watch?v=e7jaz5SFvFk
}

\begin{abstract}
There are numerous barriers to the use of scientific computing toolsets. These barriers are becoming more apparent as we increasingly see mixing of different academic backgrounds, and compute ranging from laptops to cloud platforms. Members of the UC Berkeley D-Lab, Statistical Computing Facility (SCF), and Berkeley Research Computing (BRC) support such use-cases, and have developed strategies that reduce the pain points that arise. We begin by describing the variety of concrete training and research use-cases in which our strategy might increase accessibility, productivity, reuse, and reproducibility. We then introduce available tools for the "recipe-based" creation of compute environments, attempting to demystify and provide a framework for thinking about DevOps (along with explaining what "DevOps" means!). As a counterpoint to novel DevOps tools, we'll also examine the success of OSGeo-Live [OSGL] - a project that has managed to obtain and manage developer contributions for a large number of geospatial projects. This is enabled through the use of commonly known skills like shell scripting, and is a model of complexity that can be managed without these more recent DevOps tools. Given our evaluation of a variety of technologies and use-cases, we present our current strategy for constructing the Berkeley Common Environment [BCE], along with general recommendations for building environments for your own use-cases.
\end{abstract}

Index Terms—education, reproducibility, virtualization

\section{Introduction}

Most readers of this paper will have dealt with the challenges of sharing or using complex compute stacks - be that in the course of instruction, collaboration, or shipping professional software. Here, we suggest an approach for introducing novices to new software that reduces complexity by providing a standard reference enduser environment. We'll discuss approaches to building and using a common environment from any major OS, including an overview of the tools available to make this easier. This approach can make it easier to provide complete and robust instructions, and make it easier for students to follow demos.

At a university, students often need to reproduce an environment required to run the software for a course. Researchers need to reproduce their collaborator's workflows, or anyone's workflow in the name of reproducible research. Recently, a new crop of tools-for-managing-tools has emerged under the DevOps banner - a contraction of software development and systems operation

* Corresponding author: davclark@berkeley.edu

+UC Berkeley

§ OSGeo California Chapter

Copyright $(2014$ Dav Clark et al. This is an open-access article distributed under the terms of the Creative Commons Attribution License, which permits unrestricted use, distribution, and reproduction in any medium, provided the original author and source are credited.
- with a general philosophy that instead of merely documenting systems operation tasks (configuration, deployment, maintenance, etc.), that developers can and should be scripting these tasks as much as possible.

In scientific computing the environment was commonly managed via Makefiles \& Unix-y hacks, or alternatively with monolithic software like Matlab. More recently, centralized package management has provided curated tools that work well together. But as more and more essential functionality is built out across a variety of systems and languages, the value - and also the difficulty - of coordinating multiple tools continues to increase. Whether we are producing research results or web services, it is becoming increasingly essential to set up new languages, libraries, databases, and more.

Documentation for complex software environments is stuck between two opposing demands. To make things easier on novice users, documentation must explain details relevant to factors like different operating systems. Alternatively, to save time writing and updating documentation, developers like to abstract over such details. A DevOps approach to "documenting" an application might consist of providing brief descriptions of various install paths, along with scripts or "recipes" that automate setup. This can be more enjoyable and certainly easily and robustly reproducible for end-users - even if your setup instructions are wrong, they will be reproducibly wrong! As we'll describe below, many readers will already have tools and skills to do this, in the form of package management and basic shell scripting. In other words, the primary shift that's required is not one of new tooling, as most developers already have the basic tooling they need. Rather, the needed shift is one of philosophy.

We recognize that excellent tools have been developed to allow for configuring Python environments, including environments that peacefully co-exist on the same computer (e.g., pip, virtualenv, venv, conda, and buildout). These specialized tools can increase our efficiency and provide ready access to a broader range of options (such as different versions or compile-time settings). But, we may also wish to coordinate the desktop environment, including text editors, version control systems, and so on. As such, these tools from the Python community to manage packages and run-time environments cannot solve all of our problems. But any of them could be used within the broader approach we'll describe.

More recent configuration management tools are directed at solving this larger problem of configuring nearly any aspect of a compute system, and yet other DevOps tools provide efficient ways of managing environments across compute contexts. Unfor- 
tunately, the variety and complexity of tools match the variety and complexity of the problem space, and the target space for most of them was not scientific computing. Thus, before discussing available tooling, we first lay out a fuller set of concerns relevant to supporting scientific computing.

\section{Issues for Scientific Computing}

The users of computational tools (and their collaborators) are often equipped with a suite of informally learned foundational skills (command line usage, knowledge of specific applications, etc.). Newcomers to a field often lack these technical skills, which creates a boundary between those who do and do not (and perhaps cannot) participate in that discipline. However, we are entering an era where these boundaries are becoming barriers to the research and educational mission of our university. Our primary concern at present for the Berkeley Common Environment [BCE] is educational, particularly introductory computational science and statistics. However, where possible, we wish to build an environment that supports the broader set of uses we outline here.

\section{For instruction}

We are entering an era where experimental philosophers want to take courses in advanced statistics and sociologists need bestof-breed text analysis. These students are willing to work hard, and might sign up for the university courses meant to provide these skills. But while the group that the course was originally designed for (e.g., statistics or computer science students) have a set of assumed skills that are necessary to succeed in the class, these skills aren't taught anywhere in the curriculum. In these cases, instructors may spend a large amount of time addressing installation and setup issues - taking time away from higher value instruction. Alternatively, students with divergent backgrounds often drop these classes with the sense that they simply can't obtain these skills. This is not an equitable situation.

It's difficult, however, to write instructions that work for any potential student. As mentioned above, students come to a course with many possible environments (i.e., on their laptop or a server). But if a standardized environment is provided, this task becomes much simpler. Written instructions need fewer special cases, and illustrations can be essentially pixel-identical to what students should be seeing on their screen.

The most accessible instructions will only require skills possessed by the broadest number of people. In particular, many potential students are not yet fluent with notions of package management, scripting, or even the basic idea of command-line interfaces [SWC]. Thus, installing an accessible solution should require only GUI operations. The installed common environment, then, can look and operate in a uniform way. This uniformity can scaffold students' use of more challenging "developer" tools. This "uniformity of the environment in which the user is clicking" cannot be implemented without full control of the graphical environment, and systems that configure only a self-contained set of libraries or computational tools cannot do this. At the other end, it would be unreasonable to reconfigure students' desktop on their laptop. Thus, we wish to set up an isolated, uniform environment in its totality where instructions can provide essentially pixelidentical guides to what the student will see on their own screen.

\section{For scientific collaboration}

Across campus, we encounter increasing numbers of researchers who wish to borrow techniques from other researchers. These researchers often come from different domains with different standards for tools. These would-be collaborators are increasingly moving towards open-source tools - often developed in Python or $\mathrm{R}$ - which already dramatically reduces financial barriers to collaboration.

The current situation, however, results in chaos, misery, and the gnashing of teeth. It is common to encounter a researcher with three or more Python distributions installed on their machine, and this user will have no idea how to manage their command-line path, or which packages are installed where. In particularly pathological cases, pip will install packages to an otherwise inactive python distribution. These nascent scientific coders will have at various points had a working system for a particular task, and often arrive at a state in which nothing seems to work. A standard environment can eliminate this confusion, and if needed, isolate environments that serve different projects. Snapshots of working systems can provide even more resilience of the continued functioning of already running projects. And it bears repeating that we don't want to disrupt the already productive environments that these researchers are using!

This issue becomes even more pronounced when researchers attempt to reproduce published results without access to the expert who did the initial research. It is unreasonable to expect any researcher to develop code along with instructions on how to run that code on any potential environment. As with the instructional case above, an easy way to do this is to ensure others have access to the exact environment the original researcher was working on, and again, "pixel-identical" instructions can be provided.

\section{For administration}

At UC Berkeley, the D-Lab supports tools for courses and short trainings. Similarly, the Statistical Computing Facility (SCF) supports an instructional lab and "cloud" resources for some courses, and grad student assistants often provide virtual machines for computer science courses (we'll explain virtual machines later). In each and every case, multiple technical challenges are common. These technical glitches can delay or reduce the quality of instruction as compared to an environment that students are already familiar with. It is also a drag on the time of those supporting the course - time that could be better directed at course content!

The more broadly a standard environment is adopted across campus, the more familiar it will be to all students. Using infrastructure for collaborative administration, technical glitches can be tracked or resolved by a community of competent contributors, allowing course instructors to simply use a well-polished end product, while reducing the complexity of instructions for students to set up course-specific software. These environments can also be tuned in ways that would be beyond the scope of what's worth doing for an individual course - for example optimizations to increase the efficiency of numeric computations or network bandwidth for remote desktops.

At this point that our use case starts to sound like the case in which product developers are working together to deploy software on a production server, while maintaining a useful development environment on their own machines, testing servers, and so on. However, going forwards, we will suggest that novel tools for building and managing compute environments be largely the domain of specialized administrator-contributors to a common environment. Technically skilled students, professors and researchers can continue to use the tools they are familiar with, such as the Ubuntu package manager, pip, shell scripts, and so on. 


\section{Technical challenges for a common environment}

Any common environment needs to provide a base of generally useful software, and it should be clear how it was installed and configured. It should be equally clear how one could set up additional software following the pattern of the "recipe" for the environment, making it easy to share new software with other users of the environment. More generally, we seek to address the following challenges, though we have not definitely solved them! After each problem, we list relevant tools, which will be described in full in a later section.

\section{Complex requirements}

The quote at the beginning of this paper illustrates a case in which requirements are not explicitly stated and there is an assumption that all collaborators know how to set up the necessary environment. The number of steps or the time required is unknown, and regularly exceeds the time available. For example, in the context of a 1.5 hour workshop or a class with only handful of participants, if all cannot be set up within a fixed amount of time (typically 20 minutes at most) it will jeopardize successfully completing the workshop or class materials and will discourage participation. All participants must be able to successfully complete the installation with a fixed number of well-known steps across all platforms within a fixed amount of time.

Additional difficulties arises when users are using different versions of the "same" software. For example, Git Bash on Windows lacks a man command. We can't control the base environment that users will have on their laptop or workstation, nor do we wish to! A useful environment should provide consistency and not depend on or interfere with users' existing setup. Relevant tools discussed below include Linux, virtual machines, and configuration management.

\section{Going beyond the laptop}

Laptops are widely used across the research and teaching space and in our experience it is reasonable to assume most individuals will have at least a 64-bit laptop with 4GB of RAM. Such a laptop is sufficient for many tasks, however the algorithms or size of in-memory data may exceed the available memory of this unitof-compute and the participant may need to migrate to another compute resource such as a powerful workstation with $128 \mathrm{~GB}$ of RAM (even the most advanced laptops typically max-out at 16GB at the time of this writing). Thus, an environment should not be restricted to personal computers. Across systems, a user should be able to to replicate the data processing, transformations, and analysis steps they ran on their laptop in this new environment, but with better performance. Relevant tools discussed below include Packer and Docker.

\section{Managing cost / maximizing value}

Imagine you have the grant money to buy a large workstation with lots of memory and many processors, but you may only need that resource for a 1 to 2 week period of time. Spending your money on a resource that remains unused $95 \%$ of the time is a waste of your grant money! A homogeneous, familiar environment can enable easier usage of the public cloud. A private cloud approach to managing owned resources can also allow more researchers to get value out of those resources. This is a critical enabler to allow us to serve less well-funded researchers. In addition, more recent technologies can avoid exclusively reserving system resources for

\begin{tabular}{|c|c|}
\hline Goal & Relevant tools \\
\hline $\begin{array}{l}\text { Make Linux available as a } \\
\text { VM (regardless of host OS) }\end{array}$ & $\begin{array}{l}\text { Local VM tool or public cloud (e.g., } \\
\text { VirtualBox or Amazon EC } 2 \text { - choose } \\
\text { something supported by Packer) }\end{array}$ \\
\hline $\begin{array}{l}\text { Apply configurations in a re- } \\
\text { peatable fashion }\end{array}$ & $\begin{array}{l}\text { Scripting, package managers (e.g., apt, } \\
\text { pip), configuration management (e.g., } \\
\text { Ansible) }\end{array}$ \\
\hline $\begin{array}{l}\text { Generate OS image for mul- } \\
\text { tiple platforms }\end{array}$ & Packer \\
\hline $\begin{array}{l}\text { Enable light-weight cus- } \\
\text { tom environment (instead of } \\
\text { heavy-weight virtualization) }\end{array}$ & Docker, LXC \\
\hline
\end{tabular}

TABLE 1: Recommended automation tools for our use-cases.

a single environment. Relevant tools discussed below are Packer, Docker (and LXC), and cloud-based virtual machines.

\section{Existing Tools}

As discussed above, the problems outlined above are not unique to scientific computing. Developers and administrators have produced a variety of tools that make it easier to ensure consistent environments across all kinds of infrastructure, ranging from a slice of your personal laptop, to a dynamically provisioned slice of your hybrid public/private cloud. We cannot cover the breadth of tooling available here, and so we will restrict ourselves to focusing on those tools that we've found useful to automate the steps that come before you start doing science. We'll also discuss popular tools we've found to add more complexity for our use-cases than they eliminate. Table 1 provides an overview from the perspective of the DevOps engineer (i.e., contributor, maintainer, you, etc.).

\section{Linux OS (Operating System)}

A foundational tool for our approach is the Linux operating system. It is far easier to standardize on a single OS instead of trying to manage cross-platform support. It is relatively easy to install (or build) scientific code and DevOps tools on Linux. Moreover, Linux is not encumbered by licensing constraints, which reduces barriers to collaboration, distribution, and reuse. This choice of a single target OS is a primary reason to use virtual machines (described below) because most people don't use Linux as their primary laptop OS.

\section{Virtual machines (VMs)}

Virtual machine (VM) software enables running another OS (in BCE, Ubuntu server with XFCE installed) as a guest OS inside the host OS - often Mac OS or Windows. If a system is not virtualized (for example, the host OS), it is said to be running on "bare metal." For BCE, we have focused on VirtualBox and VMware (the former of which is free) as they both run on Windows, Mac OS, and Linux. Cloud providers like EC2 only provide virtual machines (there is no access to "bare metal"), and similar concepts apply across local and cloud virtual systems. A notable distinction is that web tools are often available for cloud services, as opposed to a local GUI tool for systems like VirtualBox. Both kinds of services provide command-line tools that can perform a superset of the tasks possible with graphical interfaces.

For some users, a VM simply will not run locally, generally because they have a very old operating system or computer. Thus, one should assume that any VM solution will not work 
for some individuals and provide a fallback solution (particularly for instructional environments) on a remote server. In this case, remote desktop software may be necessary, or in the case of $\mathrm{BCE}$, we are able to enable all essential functionality via a web browser using IPython notebooks. RStudio server would provide a similar approach to sidestepping the need for a full remote desktop session.

One concern is that VMs reserve compute resources exclusively. Some approaches, however, allow for more elastic usage of resources, most notably with LXC-like solutions, discussed in the Docker section below. Another issue that can arise is dealing with mappings between host and guest OS, which vary from system to system - arguing for the utility of an abstraction layer for VM configuration like Vagrant or Packer (discussed below). This includes things like port-mapping, shared files, enabling control of the display for a GUI vs. enabling network routing for remote operation. These settings may also interact with the way the guest OS is configured. Specifically with BCE we noticed that some desktop environments interacted poorly with VirtualBox (for example, LXDE did not handle resize events properly).

Note that if you are already running Linux on "bare metal", it's still useful to run a virtualized Linux guest OS. The BCE model relies on a well-known, curated set of dependencies and default configurations. To ensure that it is possible to consistently and reliably manage those elements no matter what flavor, variant, or version of Linux you may be running as the host OS. However, we have intentionally made choices that allow an informed developer set up a partial environment that matches BCE. For example, python requirements are installed with pip using a requirements file. This makes it easy to set up a virtualenv or conda environment with those packages.

The easiest way to use a VM is to use a pre-existing image - a file that contains all relevant data and metadata about an environment (described more fully at [images]). It's very easy to make modifications to an environment and make a new image by taking a snapshot. Note that while both local and cloud-based VM systems often allow for easy snapshotting, it may be hard to capture exactly how changes happened - especially changes and configuration that was made "by hand." So, snapshots are not necessarily a good solution for reproducibility. You can also install an OS to a virtual image in essentially the same manner you would install it to bare metal. The primary difference is that you need to use specialized VM software to start this process. For example, you can do this directly in VirtualBox simply by clicking the "New" button, and you'll be guided through all of the steps. There are more automated ways, however, and we discuss these below.

\section{Configuration management and automated image creation}

Creating an image or environment is often called provisioning. The way this was done in traditional systems operation was interactively, perhaps using a hybrid of GUI, networked, and command-line tools. The DevOps philosophy encourages that we accomplish as much as possible with scripts (ideally checked into version control!). Most readers of this paper will already be able to create a list of shell commands in a file and execute it as a script. So, if you already know how to execute commands at the Bash prompt to configure Linux, this can do most of the system setup for you.

Package managers in particular provide high-level commands to install and configure packages. Currently, we use a combination of apt, pip, and shell scripts. We also evaluated conda and found that it introduced additional complexity. For example, it is still hard to install a list of pip requirements with conda if some packages are not available for conda. Most package authors currently make their packages available, however, for pip. Standard apt packages were also adequate for things like databases, and ideal for the desktop environment, where we could reap the benefit of the careful work that went into the LTS Ubuntu distribution.

Some steps may even be done manually. As we explored managing the complexity and reducing the number of tools for the BCE development process, one of the steps in the "recipe" was manual installation of Ubuntu from an ISO. It is straightforward to make a binary image from a snapshot immediately after creating a base image, so this initial step could be done once by a careful individual.

Ultimately, however, we decided it was better to automate installation from an ISO, which is enabled by the Debian Installer [UDI], a system that allows a text file to specify answers to the standard configuration prompts at install-time, in addition to providing many more possibilities. You can find the BCE configuration file for the debian-installer in the provisioning/http directory. Later, we'll discuss how we're coordinating all of the above using Packer.

\section{Ansible and related tools}

Ansible is one of a number of recent DevOps tools for configuration management [Ansible]. These tools enable automated management of customizations to the default status and configuration of software. They are purpose-built domain-specific tools that can replace the scripting approach described above. Such systems provide checks and guarantees for applying changes that would be hard to write as shell scripts alone - just as a makefile handles builds more gracefully than a shell script. This approach manages configuration complexity as an environment grows in feature complexity. It may also allow an end-user to manage and reliably apply personal customizations across multiple versions of an environment over time. For BCE development, we felt Ansible added the least complexity amongst comparable tools. It may be used at build-time and also at run-time within the guest OS, or from any other location with SSH access to the target being configured. The only requirements for the target are an SSH server and a Python interpreter (Ansible is Python-based). Ansible execution is also more linear than some systems, which is a limitation, but also a simplification.

At this phase, however, the complexity of BCE doesn't warrant contributors learning even a simple configuration management tool. The maintainer of the Software Carpentry VM, Matt Davis, has reported a similar observation. He has used another tool, Puppet, to provision the Software Carpentry VM, but will likely use shell scripts in the future. And as we will see below from the OSGeo project, it is perhaps easier to coordinate certain kinds of complexity with more commonly known tools like shell scripting.

While the syntax for each tool varies, the general concept is the same - one describes the desired machine state with a toolspecific language. After execution of this recipe - if you did a good job - the machine state is guaranteed to be how you've requested it to be. Unfortunately, all DevOps tools call their recipes something different. While the process certainly seems more like baking than, say, coaching a football team, Ansible calls its scripts "playbooks." Alternate tools with similar functionality are Chef (which, unsurprisingly does call its scripts "recipes"), 
Salt (also Python-based, and uses "states"), and Puppet (which uses "manifests"). With any of these, a great way to start learning would be to translate an existing configuration shell script into one of these tools.

\section{Packer}

Packer is used at build-time and enables creating identical machine images targeting multiple machine image formats [Packer]. For example, we generate a (mostly) uniformly configured BCE machine image in multiple formats including OVF for VirtualBox and AMI for AWS EC2. Packer coordinates many of the tools described above and below based on a JSON configuration file. This file specifies the Ubuntu ISO to install, a Debian Installer configuration file (which gets served over HTTP), and configures the installed OS by copying files and running a shell script. Packer can also readily use Ansible, Puppet, Chef, or Salt (and has a plugin system if you want to use something more exotic). Images can be built for many popular platforms, including a variety of local and cloud-based providers.

Packer made it possible for us to learn a relatively simple tool that executes the entire image-creation process as a single logical operation. Moreover, end users need have no knowledge of Packer. They can use the Amazon web console or the VirtualBox GUI with no concerns for the complexity at build time.

It is worth noting that while indexes are available for a variety of images (e.g, vagrantbox.es, the Docker index, and Amazon's list of AMIs), we have encountered surprisingly little effort to publish consistent environment that allows one to readily migrate between platforms. This is, however, precisely the goal of BCE, and it's enabled by Packer.

\section{Vagrant}

Vagrant is a run-time component that needs to be installed on the host OS of the end user's laptop [Vagrant]. Like Packer, it is a wrapper around virtualization software that automates the process of configuring and starting a VM from a special Vagrant box image (Vagrant boxes may be created with any of the above tools). It is an alternative to configuring the virtualization software using the GUI interface or the system-specific command line tools provided by systems like VirtualBox or Amazon. Instead, Vagrant looks for a Vagrantfile which defines the configuration, and also establishes the directory under which the vagrant command will connect to the relevant VM. This directory is, by default, synced to the guest VM, allowing the developer to edit the files with tools on their host OS. From the command-line (under this directory), the user can start, stop, or ssh into the Vagrant-managed VM. It should be noted that (again, like Packer) Vagrant does no work directly, but rather calls out to those other platform-specific command-line tools.

The initial impetus for the BCE project came from a Vagrantbased project called "jiffylab" [j1]. With a single command, this project launches a VM in VirtualBox or on various cloud services. This VM provides isolated shell and IPython notebook through your web browser. But while Vagrant is conceptually very elegant (and cool), we are not currently using it for BCE. In our evaluation, it introduced another piece of software, requiring command-line usage before students were comfortable with it. Should a usecase arise, however, it would be trivial to create a "vagrant box" (a Vagrant-tuned virtual image) with our current approach using Packer. That said, other "data-science" oriented VMs have chosen Vagrant as their method of distribution [DSTb], [DSTk].
Currently, Vagrant is most useful for experienced developers to share environments with each other.

\section{Docker}

Docker is a platform to build, distribute, and run images built on top of Linux Containers (LXC) which provides a lightweight style of virtualization called containerization [Docker]. An important distinction of LXC-based containerization is that the guest OS and the host OS both run the same underlying Linux kernel.

At run-time Docker adds to this containerization a collection of tools to manage configuring and starting an instance in much the same way that Vagrant does for a virtualization environment. Images are created using a simple build script called a Dockerfile which usually runs a series of shell script commands which might even invoke a configuration management system such as Ansible.

Another feature of the platform is the management and distribution of the images built by docker, including incremental differences between images. Docker makes it possible (albeit in a rudimentary way) to track changes to the binary image in a manner similar to the way git allows you to track changes to source code. This also includes the ability to efficiently maintain and distribute multiple branches of binary images that may be derived from a common root.

Docker is also more than just a tool. It is a quickly growing community of open source and industry developers with a rapidly evolving ecosystem of tools built on core OS primitives. There is no clear set of best practices, and those that emerge are not likely to fit all the use cases of the academic community without us being involved in mapping the tools to our needs. However, providing better access to hardware with containers is an important and active research topic for performance [HPC].

Currently, Docker requires a Linux environment to host the Docker server. As such, it clearly adds additional complexity on top of the requirement to support a virtual machine. We also evaluated Docker as a way to potentially provide around 30 students access to a VM on a reasonably powered server with only 16GB of RAM. However, in our use-cases, we have full control of our Linux compute environment and existing methods of isolating users with permissions was less complex than using Docker, and of course allowed users to efficiently share all available physical RAM. Moreover, the default method of deploying Docker (at the time of evaluation) on personal computers was with Vagrant. This approach would then also add the complexity of using Vagrant. However, recent advances with boot2docker provide something akin to a VirtualBox-only, Docker-specific replacement for Vagrant that eliminates some of this complexity, though one still needs to grapple with the cognitive load of nested virtual environments and tooling.

\section{OSGeo-Live: A Successful Common Environment}

The OSGeo-Live VM is an example of a comprehensive geospatial compute environment with a vibrant community process. It provides a successful example of solving the problems of complex requirements described above - or in this case, perhaps more properly called "dependency hell". Notably, the project uses none of the recent DevOps tools. OSGeo-Live is instead configured using simple and modular combinations of Python, Perl and shell scripts, along with clear install conventions and examples. Documentation is given high priority.

The VM project began around the same time as, and ultimately joined the Open Source Geospatial Foundation (OSGeo), 
an international body modeled on the Apache Foundation [2g]. It started as a smaller open project that sought to build an "easy to try and use" software environment for spatial data applications. Initial efforts consisted of shell scripts to install core geospatial packages. These examples provided guides to the projects that were invited and ultimately contributed packages to the project. Many of these later contributors spoke English as a second language, further highlighting the importance of clear, working code examples. OSGeo-Live is not the only attempt at building such an environment, but it is a highly successful one. More than fifty open-source projects now contribute by actively maintaining and improving their own install scripts, examples and documentation.

\section{Tool Sets}

OSGeo-Live itself is not a "Linux distribution" per se, rather it relies on an apt-based ecosystem to handle the heavy-lifting of system updates and upgrades. This is a win, as updates are proven reliable over a very large Ubuntu community process, and project participants can concentrate on adding value to its featured components. Given the component architecture used to build the VM, individual software projects can be installed as-needed on a generic apt-enabled base.

A key component of the success of the overall project has been the availability of widely-known and reliable tools. Rather than require . deb installation packages for each project, OSGeo-Live chose to use a simple install script format, with ample examples. This choice proved crucial in the earliest stages, as an outside open-source project evaluating participation in the Live ISO could get started with fewer barriers to entry. Participating open-source projects already had install scripts built for Linux, so they could almost immediately adapt and iterate their own install scripts in a straightforward way, with the flexibility to use the tools they were already using, such as shell, Perl, or Python. Scripts may call package managers, and generally have few constraints (apart from conventions like keeping recipes contained to a particular directory). The project also maintains packages that support broader kinds of packages, such as web-based applications. In this case, OSGeo-Live provides a standard configuration for Apache, WSGI, and other components, along with a standard layout for projects that rely on this core. As a result, there is very little conflict among packages that share common resources. Some concerns, like port number usage, have to be explicitly managed at a global level. But the overhead of getting 50 projects to adopt a uniform configuration management tool would likely be much greater.

All recipes are currently maintained in a common subversion repository, using standardized asset hierarchies, including installation scripts [6g]. An OSGeo-Live specific report is maintained on the project trac ticketing system [10g]. And while OSGeoLive primarily targets a live/bootable ISO, the scripts that are used to build that ISO provide a straightforward method for building OSGeo software in other contexts.

\section{Community Awareness}

The initial stages of the adoption of new technology include initial awareness and trialability [4g]. OSGeo-Live intentionally incorporates targeted outreach, professional graphic design and "easy to try" structure to build participation from both developers and end-users. An original project design goal was to provide tools to those doing geospatial fieldwork with limited resources around the globe, and who often lack advanced programming and administration skills. In other words, a community was built around tools that the desired members already had.

Several years into the project, with a grant from the Australian government, a professional-level documentation project was initiated for a single-page overview and quick-start instructions for each application. Language internationalization was rendered more efficient, specifically to support local field work. Much later, a "percentage complete" graph for each human language group was added, making translation into a sort of competitive game. This translation has proven very successful. The project has facilitated collaboration across developer communities. For example, we have seen productive application of software developed by the U.S. military to environmental applications [Army].

\section{Steps to Contribute}

All build scripts are organized in the open, in source control [6g]. A new contributors FAQ is maintained via wiki [7g] for software projects, and for translation [8g]. At its core, the OSGeoLive project uses common skills for system administration as opposed to more recent DevOps available, but it very much adopts a DevOps philosophy. Contributors pay particular attention to documenting each and every step, and standard approaches are encouraged across the project. Gamification also played a role in spurring useful documentation contributions. The low barrier to entry (allowing contributing projects to use skills they likely already have), combined with guidelines to ensure interoperability have led to OSGeo-Live becoming a standard way to evaluate and install software in the geospatial community.

\section{BCE: The Berkeley Common Environment}

The overarching, aspirational goal for the Berkeley Common Environment (BCE) is to make it easy to do the "right" thing (or hard to do "wrong" things), where "right" means you've managed to use someone else's code in the manner that was intended. In particular, it allows for targeted instructions that can assume all features of BCE are present. BCE also aims to be stable, reliable, and reduce complexity more than it increases it.

More prosaically, to be useful in the cases described above, BCE provides simple things like a standard GUI text editor, and a command-line editor for when a GUI is not available. BCE preconfigures applications with sensible defaults (e.g., spaces for tabstops are set up for nano). It also enables idiosyncratic features on different VM platforms, for example, enabling simple access to shared folders in VirtualBox and ensuring NFS functions properly on Amazon EC2. The environment is also configured to make minimal demands on underlying resources. For example, the BCE desktop is a solid color to minimize network utilization for remote desktop sessions, and efficient numerics libraries are configured.

BCE provides ready-made images for end-users, and the "recipe" for setting up the image using Packer is maintained on GitHub. Lists of Python packages are maintained in a separate requirements file, and all setup is done via a master Bash script. It is currently common for individuals to only distribute scripts, which requires all potential users to install and configure the relevant stack of DevOps tools. There are, however, free services for distributing images for particular tools (e.g., the Docker index), and services like Amazon can host AMIs for pennies a month. (For example, building on a free, existing EBS-backed AMI, one need only save a snapshot, with charges only for changes from the base AMI. One GB of extra tools onto a standard EBS-backed 


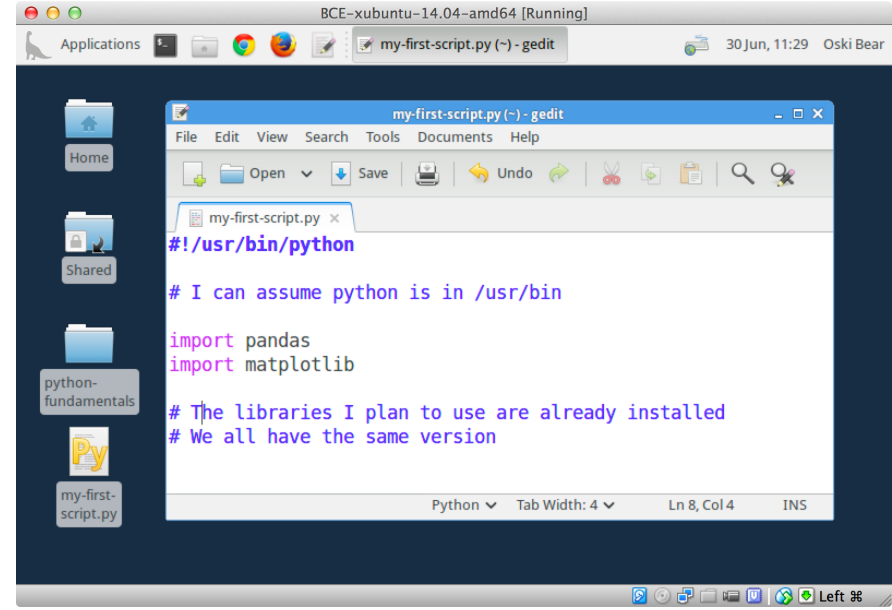

Fig. 1: The Berkeley Common Environment running in VirtualBox on OS X. The interface (and opportunities for confusion) are minimized. For example, all users have the same text editor available, and in particular, it's easy to configure common gotchas like spaces for tabs.

Ubuntu server AMI, currently costs $<\$ 0.1$ / GB-month to store.) We strongly recommend distributing a binary along with the recipe for any environment that includes novices in its audience.

\section{Using the $B C E$}

You can see what BCE currently looks like (in a relatively small window) in Figure 1. Throughout various iterations, students have found working on a BCE VM to be confusing and counterproductive to being incredibly useful and efficient - strong evidence that the details matter. It seems critical both to provide a rationale for the use of VMs (i.e., explaining how a standard, "pixel-identical" environment speeds instruction), and also a smooth initial experience. Thus, we've worked to make BCE easy for students, researchers, and instructors. Simple instructions are provided on our site for things like opening a terminal (including a description of what the terminal icon looks like). However, for an experienced programmer, the environment should be obvious to navigate.

In our experience, some students will not be able to run the VM while others have difficulty getting regular access to a stable network connection (though fortunately, almost never both!). So, consistency across server and local versions of the environment is critical to effectively support students with either of these difficulties.

If you're using VirtualBox, we require a 64-bit CPU with support for 64-bit virtualization (note that some 32-bit operating systems will support this on some hardware). A reasonable minimum of RAM is 4GB. The full instructions for importing BCE from an OVA image into Virtualbox are available on our project website [BCEVB]. After starting the $\mathrm{VM}-$ a process that can be done entirely with the mouse - a user will have all the software installed as part of BCE, including IPython, RStudio, and useful packages.

If you're using BCE on EC2, even a micro instance is sufficient for basic tasks. Again, complete instructions are provided on the BCE website [BCEAMI]. In brief, you can find our image (AMI) in the public list. You can readily launch in instance, and get instructions on connecting via the EC2 console.

\section{Communicating with the maintainers of the BCE project}

All development occurs in the open in our GitHub repository. This repository currently also hosts the project website, with links to all BCE materials. We provide channels for communication on bugs, desired features, and the like via the repository and a mailing list (also linked from the project page), or if a user is comfortable with it, via the GitHub issue tracker. BCE will be clearly versioned for each semester, and versions will not be modified, except for potential bugfix releases.

\section{Contributing to the BCE project}

BCE provides a fully scripted (thus, reproducible) workflow that creates the standard VM/image. If the appropriate software is installed, the recipe should run reliably. However, you should generally not need to build the binary VM for BCE for a given semester. If you wish to customize or extend BCE, the best way to do this is by simply writing a shell script that will install requirements properly in the context of BCE (for a complex example, see our bootstrap-bce.sh script [boot]). Much as with OSGeo-Live, we have chosen our approach to provisioning to be relatively simple for users to understand. It is our goal for instructors or domain experts to be able to easily extend the recipe for building BCE VMs or images. If not, that's a bug!

As described above, while we have experimented with Docker, Vagrant, and Ansible for setting up the various BCE images (and evaluated even more tools), the only foundationally useful tool for our current set of problems has been Packer. Packer runs a shell script that uses standard installation mechanisms like pip and apt-get to complete the setup of our environment. Of central importance, Packer does not require end-users to install or understand any of the current crop of DevOps tools - it operates solely at build time. However, should the need arise, Packer will readily target Vagrant, Docker, and many other targets, and we are not opposed to adopting other tooling.

\section{Conclusion}

By merely using recent DevOps tools, you arrive at the cutting edge of DevOps for the scientific community. Your collaborators and students likely won't have needed concepts, so extra care should be taken to make your tooling accessible. Where appropriate, use tools that your collaborators already know - shell, scripting, package management, etc. That said, technologies that allow efficient usage of available hardware, like Docker, stand to provide substantial savings and potential for re-use by researchers with less direct access to capital.

So, let's be intentional about creating and using environments that are broadly accessible. Let's follow the DevOps philosophy of being transparent and explicit about our choices and assumptions. That doesn't have to mean "using the latest tools" - a simple text file or even a PDF can provide ample explanation that a human can understand, along with a simple reference script (in shell or Python). In this paper, we've made fairly strong recommendations based on what we are actually using (we are eating our own dogfood!). A novice user can access BCE using only a few GUI operations on their laptop, or the Amazon Web Console. As we've seen with OSGeo-Live, the simple tools we've chosen make it easy for our collaborators (instructors or researchers) to understand. This standard reference allows us to return focus on the interesting bits of developing code and doing science. 
BCE currently provides a standard reference, built with an easily understood recipe, that eliminates the complexity of describing how to run a large variety of projects across a wide variety of platforms. We can now target our instruction to a single platform. The environment is easy to deploy, and should provide identical results across any base platform - if this is not the case, it's a bug! This environment is already available on VirtualBox and Amazon EC2, and is straightforward to provision for other environments. We welcome loose collaboration in the form of forks that are specialized for other institutions, and eventually, perhaps standardizing across institutions.

\section{RefERENCES}

[BCE] http://collaboratool.berkeley.edu

[OSGL] http://www.osgeo.org/

[BCEVB] http://collaboratool.berkeley.edu/using-virtualbox.html

[BCEAMI] http://collaboratool.berkeley.edu/using-ec2.html

[Ubuntu] https://help.ubuntu.com/14.04/serverguide/serverguide.pdf

[images] http://docs.openstack.org/image-guide/content/ch_introduction. html

[Ansible] http://www.ansible.com/about

[Packer] http://www.packer.io/intro

[Vagrant] http://www.vagrantup.com/about.html

[Docker] http://www.docker.com/whatisdocker/

[HPC] M. G. Xavier, M. V. Neves, F. D. Rossi, T. C. Ferreto, T. Lange, and C. A. De Rose, "Performance evaluation of container-based virtualization for high performance computing environments," in the 21 st Euromicro International Conference on Parallel, Distributed and Network-Based Processing (PDP), 2013, pp. 233-240.

[SWC] G Wilson, "Software Carpentry: lessons learned," F1000Research, 2014.

[jl] http://github.com/ptone/jiffylab

[DSTb] http://datasciencetoolbox.org/

[DSTk] http://www.datasciencetoolkit.org/

[UDI] https://help.ubuntu.com/14.04/installation-guide/i386/apb.html

$[2 \mathrm{~g}] \quad \mathrm{http}: / /$ www.osgeo.org/content/foundation/about.html

[4g] E M. Rogers, Diffusion of Innovations, 5th ed. New York: Free Press, 2003.

[6g] http://svn.osgeo.org/osgeo/livedvd

[7g] http://wiki.osgeo.org/wiki/Live_GIS_Add_Project

[8g] http://wiki.osgeo.org/wiki/Live_GIS_Translate

[10g] http://trac.osgeo.org/osgeo/report/10

[Army] Army Corps of Engineers, "Army Corps of Engineers Wetlands Regulatory program," presented at the FOSS4G, 2007.

[boot] https://github.com/dlab-berkeley/collaboratool/blob/master/ provisioning/bootstrap-bce.sh 